\title{
Estimating 3-D Body Parameters from Reflection Component Separating Imagery: A Performance Analysis
}

\author{
J.C. Glas, F. van der Heijden
}

\begin{abstract}
Iterative least-squares estimation requires accurate reflectance models to retrieve geometrical parameters of 3-D objects from an image projection. We investigate the use of separating the diffuse (body) reflection from the specular (surface) reflection, where the latter is responsible for image highlights. The performance of several models has been analysed by comparing local higher-order derivatives of the least-squares error function. Experiments show that the (smooth) diffuse component yields the best convergence properties, while the (sharp) specular component can be utilized to improve noise insensitivity.
\end{abstract}

\section{Introduction}

Retrieving geometrical parameters of 3-D objects in a scene from a 2-D image projection is a non-linear inverse problem for which iterative least-squares estimation yields an optimal solution provided that the measurement noise obeys an additive Gaussian model. The method requires prior knowledge about light, material and camera properties applied in accurate imaging and reflectance models. Estimates of the parameters to be retrieved are passed to these models to generate an image prediction that will be compared with the real image. Local linearization of the model function settles the least-squares difference into an update of the parameter estimates. This procedure is repeated until the difference reaches a minimum. Korsten [1], De Graaf et al [2] and Lowe [3] have all demonstrated that such a model based approach to image understanding yields good results in estimating 3-D object parameters from camera pictures.

The selection of reflectance models, imaging models and other submodels of the total measurement model will affect the performance of the parameter estimation process. The overall performance is determined by convergence speed and area and by insensitivity to measurement noise. The estimator provides local minimization of the least-squares error between real and predicted measurements. While the first-order partial derivatives of the error function are zero at the point of convergence, the second-order partial derivatives at that place indicate the steepness increase of the error function in the direct neigbourhood of the final

\footnotetext{
${ }^{*}$ Department of Electrical Engineering, Control, Systems and Computer Engineering Group, Laboratory for Measurement Science and Instrumentation, P.O. Box 217, 7500 AE Enschede, The Netherlands, E-mail: jaap@mi.el.utwente.nl
}

\author{
University of Twente* ${ }^{*} \mathrm{NL}$
}

estimate. A steeper error function implies a smaller deviation in parameter values at the same deviation in the measurements. Therefore, local second-order derivatives of the error function can be used to assess the noise insensitivity of the measurement model.

Differences in the convergence properties of the estimation process are mainly depending on the measure of linearity of the model function. Increasing non-linearity of the model will increase the number of iterations required to converge, because the iterative estimator is driven by local linearization of the model function. The non-linearity annuls the constancy of the second-order partial derivatives of the least-squares error, which is quantified by the magnitude of the third-order partial derivatives. Therefore, the local third-order derivatives of the error function can be used to measure the convergence speed of the iterative least-squares estimator in the neigbourhood of the point of convergence.

The use of local derivatives of the error function enables us to analyse the convergence speed and insensitivity to measurement noise of our estimation problems without the experimental effort of running the iterative estimator for many different initial estimates and noise realizations. Furthermore, the analysis may be restricted to a limited number of points in parameter space that are considered to be representative for the estimation problems that have to be solved. Analysis of the local derivatives in these points offers the possibility to compare different (sub)models and select the one with the best performance. We applied this method to compose our reflectance model.

\subsection{Reflection component separation}

The reflectance from the surface of an object can be considered as a sum of diffuse (body) reflection and specular (surface) reflection (see e.g. Nayar et al [11]). The former component can be modelled by the wellknown Lambertian reflectance model. The Torranceand-Sparrow model [12] for (off)-specular reflection of rough surfaces has been applied to model the latter component. It accounts for the occurrence of shiny spots upon curved surfaces. We consider these so called highlights to be useful clues for shape from shading rather than inconvenient image disturbances. Figure 1 shows the image irradiance profile of such a specularity taken by a line-scan camera.

The sharpness of the specular reflection component increases the steepness of the error function which im- 


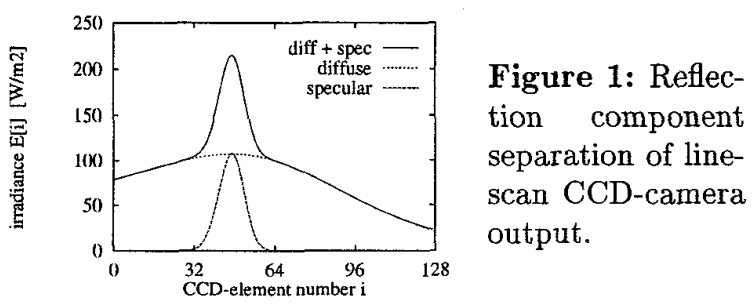

plies that the measurement model should become less sensitive to noise. Compared to the smoothness of the diffuse reflection model however, that same sharpness is also responsible for a significant increase in the non-linearity of the model function. It would explain the convergence problems of the estimator as experienced while taking measurements directly from an image that contains highlights. This duality in the usefulness of highlighted images to the estimation process leads to the hypothesis that separating diffuse and specular reflection components in advance may benefit the overall performance of the estimator.

Inherent differences in the spectral distribution and/or polarization of diffuse and specular reflectance offer possibilities to separate reflection components in images that contain highlights. The spectral density of the diffuse component is the product of the spectral densities of the light source and the surface reflectance, while the spectral density of the specular component originates from the light source only. Gershon [4] and Klinker [5] utilize this distinction to separate reflection components in colour images. Wolff and Boult [6] demonstrated how the unpolarized diffuse reflectance can be separated from the partially polarized specular reflectance. Nayar et al [7] have integrated both separation methods.

Our paper tentatively assumes that the separation between the diffuse and specular reflection components can be established. Figure 1 shows the result of this operation on the output of a line-scan camera. We concentrate on the use of separated reflection components to the convergence properties and noise insensitivity of estimating geometrical parameters of 3-D objects from a highlighted image. A local derivatives comparison of reflection models has been performed to demonstrate that the best convergence is obtained from estimation with the (smooth) diffuse component only, but that final assistance of the (sharp) specular component improves the accuracy of the estimates.

\section{Parameter estimation}

The parameter estimation of 3-D objects from a 2-D image is performed by a general method to determine an optimal estimate of an unknown parameter vector $\vec{\alpha}$ from a measurement vector $\vec{\theta}$ given the forward model

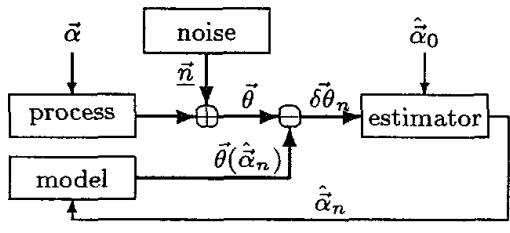

Figure 2: Iterative parameter estimation scheme.

$\vec{\theta}(\vec{\alpha})$ of the non-linear relationship between them:

$$
\vec{\theta}=\vec{\theta}(\vec{\alpha})+\underline{\vec{n}}
$$

The notation has been adopted from Korsten [1]. In case the additive measurement noise $\underline{\overrightarrow{ }}$ has a zero-mean Gaussian distribution and prior knowledge about $\vec{\alpha}$ is not available, the maximum a posteriori estimate of $\vec{\alpha}$ results from minimization of the weighted leastsquares error $\ell(\vec{\alpha})$ of the measurement vector:

$$
\ell(\vec{\alpha})=\frac{1}{2}(\vec{\theta}-\vec{\theta}(\vec{\alpha}))^{T} C_{\vec{\theta}}^{-1}(\vec{\theta}-\vec{\theta}(\vec{\alpha}))
$$

The weighting matrix is given by the inverse of the measurement covariance matrix $C_{\vec{\theta}}$, which equals the noise covariance $C_{\overrightarrow{\underline{n}}}$.

\subsection{The Gauss-Newton method}

If the model is piecewise differentiable, the GaussNewton method (see e.g. Sorensen [8]) can be applied to minimize Eq. (2) by iterative linearization around the previous estimate $\hat{\vec{\alpha}}_{n}$ of parameter vector $\vec{\alpha}$. Linearizing $\vec{\theta}(\vec{\alpha})$ from Eq. (1) results in the following measurement error vector $\overrightarrow{\delta \theta}_{n}$ :

$$
\overrightarrow{\delta \theta} \vec{\theta}_{n}=\vec{\theta}-\vec{\theta}\left(\hat{\vec{\alpha}}_{n}\right)=\left.\frac{\partial \vec{\theta}(\vec{\alpha})}{\partial \vec{\alpha}}\right|_{\vec{\alpha}=\hat{\vec{\alpha}}_{n}}\left(\vec{\alpha}-\hat{\vec{\alpha}}_{n}\right)+\underline{\vec{n}}
$$

The first-order partial derivatives of model $\vec{\theta}(\vec{\alpha})$ around $\vec{\alpha}_{n}$ form a Jacobian matrix $B_{\hat{\vec{\alpha}}_{n}}$, whose numerical approximation $B_{\vec{\Delta}, \hat{\tilde{\alpha}}_{n}}$ may be defined as follows:

$$
B_{\vec{\Delta}, \hat{\vec{\alpha}}_{n}}=\left(\ldots \frac{\vec{\theta}\left(\hat{\vec{\alpha}}_{n}+\Delta_{i} \vec{e}_{i}\right)-\vec{\theta}\left(\hat{\vec{\alpha}}_{n}\right)}{\Delta_{i}} \ldots\right)_{i=1 \ldots m}
$$

Elements $\Delta_{1}$ to $\Delta_{m}$ of vector $\vec{\Delta}$ contain the finite differences of the $m$ parameters to estimate and $\vec{e}_{1}$ to $\vec{e}_{m}$ are the unit vectors of parameter space.

If the prior uncertainty about parameter vector $\vec{\alpha}$ is expressed by an infinite covariance $C_{\vec{\alpha}}=\infty I$, an unbiased weighted least-squares estimator can be derived for the parameter error vector $\overrightarrow{\delta \alpha_{n}}$ from Eq. (3):

$$
\hat{\overrightarrow{\delta \alpha}}{ }_{n}=\vec{\alpha}-\hat{\vec{\alpha}}_{n}=\left(B_{\hat{\vec{\alpha}}_{n}}^{T} C_{\vec{\theta}}^{-1} B_{\hat{\vec{\alpha}}_{n}}\right)^{-1} B_{\hat{\vec{\alpha}}_{n}}^{T} C_{\vec{\theta}}^{-1} \overrightarrow{\theta \theta}_{n}
$$

In case of uncorrelated measurement noise, covariance matrix $C_{\vec{\theta}}^{-1}$ is diagonal and may be ignored. The posterior covariance matrix $C_{\vec{\delta} \hat{\alpha}_{n}}$ which equals the parameter covariance $C_{\hat{\vec{\alpha}}_{n}}$ is given by:

$$
C_{\hat{\delta} \alpha_{n}}=\left(B_{\hat{\tilde{\alpha}}_{n}}^{T} C_{\vec{\theta}}^{-1} B_{\hat{\vec{\alpha}}_{n}}\right)^{-1}=C_{\hat{\vec{\alpha}}_{n}}
$$

Iterative application of Eq. (5) yields the GaussNewton method. Figure 2 shows a schematic overview. 

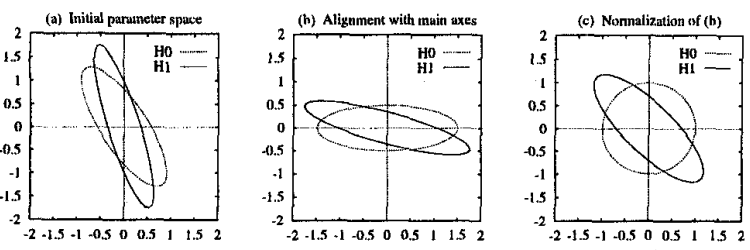

Figure 3: Transform $H_{1}$ to a metric defined by $H_{0}$.

\section{Performance analysis}

Local higher-order derivatives of the least-squares error function were recommended for measuring parameter estimation performance. Tarantola [9] derives the following Hessian matrix which contains the secondorder partial derivatives of error function $\ell(\vec{\alpha})$ as defined in Eq. (2):

$$
\frac{\partial^{2} \ell(\vec{\alpha})}{\partial \vec{\alpha}^{2}}=\frac{\partial \vec{\theta}(\vec{\alpha})}{\partial \vec{\alpha}} C_{\vec{\theta}}^{T} \frac{\partial \vec{\theta}(\vec{\alpha})}{\partial \vec{\alpha}}-\frac{\partial^{2} \vec{\theta}(\vec{\alpha})}{\partial \vec{\alpha}^{2}} C_{\vec{\theta}}^{-1}(\vec{\theta}-\vec{\theta}(\vec{\alpha}))
$$

Local linearization of the model assumes local secondorder derivatives of the model function $\vec{\theta}(\vec{\alpha})$ to be zero. The second term of the Hessian matrix from Eq. (7) vanishes in that case, after which rewriting the first term gives Hessian matrix $H_{\hat{\tilde{\alpha}}_{n}}$ that contains the local second-order derivatives around estimate $\hat{\vec{\alpha}}_{n}$ :

$$
H_{\hat{\tilde{\alpha}}_{n}}=B_{\hat{\tilde{\alpha}}_{n}}^{T} C_{\vec{\theta}}^{-1} B_{\hat{\tilde{\alpha}}_{n}}
$$

Matrix $H_{\hat{\hat{\alpha}}_{n}}$ is symmetric and positive-semidefinite [8]. An attending reader may have observed that $H_{\hat{\bar{\alpha}}_{n}}$ is the inverse of the posterior parameter covariance matrix $C_{\hat{\delta \hat{\alpha}_{n}}}$ from Eq. (6). Steepness increase of the error function around $\hat{\vec{\alpha}}_{n}$ is inversely proportional to the covariance of this estimate. If error vector $\overrightarrow{\delta \alpha}$ has a Gaussian distribution, its probability density is defined by the quadratic form in equation:

$$
{\overrightarrow{\delta \alpha_{n}}}_{n}^{T} H_{\hat{\vec{\alpha}}_{n}}{\overrightarrow{\delta \alpha_{n}}}_{n}={\overrightarrow{\delta \alpha_{n}}}_{n}^{T} C_{\hat{\bar{\alpha}}_{n}}^{-1} \overrightarrow{\delta \alpha_{n}}=c^{2}
$$

It decribes an error ellipsoid in parameter space around estimate $\hat{\vec{\alpha}}_{n}$. This contour of constant probability bounds the $c-\sigma$ confidence interval [8]. An example in Figure 3-(a) shows error ellipsoids of the 2-D matrices $H_{0}$ and $H_{1}$ for equal value of $c^{2}$.

\subsection{Comparing Hessian matrices}

A comparison of two (or more) Hessian matrices originating from different estimates or measurement models is not a trivial procedure, because each matrix contains $m^{2}$ partial derivatives in different directions of the $m$-dimensional parameter space. Defining an arbitrary norm on $H_{\hat{\vec{\alpha}}}$ to weigh the contributes of all partial derivatives is meaningless when dealing with a parameter space that spans parameters of different physical dimensions (e.g. meters and radians). The criterion to compare Hessian matrices has to be independent of the physical dimensions of parameter space [9]. Such a relative comparison between a pair of those matrices can be established by examining one of them on a metric defined by the other.

Representing Hessian matrix $H_{1}$ on a metric defined by Hessian matrix $H_{0}$ requires a matrix transformation that normalizes the latter one. The transition matrix of the corresponding co-ordinate transformation is given by the root $R=V \Lambda^{\frac{1}{2}}$ of matrix $H_{0}$ :

$$
H_{0}=V \Lambda V^{T}=\left(V \Lambda^{\frac{1}{2}}\right)\left(V \Lambda^{\frac{1}{2}}\right)^{T}=R R^{T}
$$

The columns of the (orthonormal) matrix $V$ are the eigenvectors of $H_{0}$, which align with the principal axes of the $H_{0}$ ellipsoid. Rotation matrix $V$ brings the axes of parameter space in coincidence with these principal axes. This first step of the co-ordinate transformation is shown in Figure 3-(b). Diagonal matrix $\Lambda^{\frac{1}{2}}$ contains the roots of the (real and positive) eigenvalues of $H_{0}$, which are the reciprocal half-lengths of the principal axes of the $H_{0}$ error ellipsoid. Matrix $\Lambda^{\frac{1}{2}}$ scales the parameter space to normalize these half-lengths. This second step of the co-ordinate transformation is shown in Figure 3-(c). The unit circle representing the $H_{0}$ ellipsoid corresponds with the unit matrix $I$ obtained by applying transition matrix $R$ from Eq. (10) in the matrix transformation of $H_{0}$ to its new metric.

$$
\left[H_{0}\right]^{H_{0}}=R^{-1} H_{0} R^{-T}=R^{-1} R R^{T} R^{-T}=I
$$

The same transformation is performed on $H_{1}$ to obtain its representation on the metric defined by $H_{0}$ :

$$
\left[H_{1}\right]^{H_{0}}=R^{-1} H_{1} R^{-T}=\Lambda^{-\frac{1}{2}} V^{T} H_{1} V \Lambda^{-\frac{1}{2}}
$$

It can be derived from this equation that matrix $\left[H_{1}\right]^{H_{0}}$ has adimensional elements. It maps a space into itself (automorphism), where Hessian matrices map dual spaces [9]. Determining the eigenvalues of $\left[H_{1}\right]^{H_{0}}$ has mathematical significance, because their independence of the physical units of parameter space guarantees a fair comparison of the Hessian matrices.

\subsection{Measuring convergence speed}

The non-linearity of the model function which mainly determines the convergence speed of the estimator was leading to inconstancy of second-order partial derivatives of the least-squares error function. The local inconstancy around an estimate $\hat{\vec{\alpha}}_{n}$ can be measured by comparing two Hessian matrices $H_{-\vec{\Delta}, \hat{\tilde{\alpha}}_{n}}$ and $H_{+\vec{\Delta}, \hat{\boldsymbol{\alpha}}_{n}}$ from neigbouring points in parameter space. These numerical approximations are obtained by evaluating Eq. (8) with numerical Jacobian matrices $B_{-\vec{\Delta}, \hat{\bar{\alpha}}_{n}}$ and $B_{+\vec{\Delta}, \hat{\alpha}_{n}}$ respectively. Clever use has been made of the possibility to vary the finite differences vector $\vec{\Delta}$ from Eq. (4). Disparities between $H_{-\vec{\Delta}, \hat{\tilde{\alpha}}_{n}}$ and $H_{+\vec{\Delta}, \hat{\tilde{\alpha}}_{n}}$ are 
representative for the third-order partial derivatives around estimate $\hat{\vec{\alpha}}_{n}$ in the directions parallel to the axes of parameter space. A fair Hessian matrix comparison will proceed in accordance with Section 3.1:

$$
\left[H_{-\vec{\Delta}, \hat{\vec{\alpha}}_{n}}\right]^{H_{+}, \vec{\Delta}, \hat{\tilde{\alpha}}_{n}}=U \Gamma_{\hat{\vec{\alpha}}_{n}} U^{T}
$$

The columns of matrix $U$ contain the eigenvectors of $H_{-\vec{\Delta}}$ on a metric defined by $H_{+\vec{\Delta}}$ and diagonal matrix $\Gamma_{\hat{\tilde{\alpha}}_{n}}$ contains the corresponding eigenvalues. Local linearity of the model function would yield equal Hessian matrices and in that case the $\left[H_{-\vec{\Delta}}\right]^{H}+\vec{\Delta}$ disparity ellipsoid coincides with the unit sphere. Therefore, the measure of non-linearity around estimate $\hat{\vec{\alpha}}_{n}$ is determined by the half-axis deviating most from unit length in a relative sense, which meets its numerical correspondence in the maximum of the eigenvalue roots $\Gamma_{i i, \hat{\vec{\alpha}}_{n}}^{\frac{1}{2}}$ and their reciprocals:

$$
l_{\hat{\vec{\alpha}}_{n}}=\max _{i=1}^{m}\left(\max \left(\Gamma_{i i, \hat{\alpha}_{n}}^{-\frac{1}{2}}, \Gamma_{i i, \hat{\alpha}_{n}}^{\frac{1}{2}}\right)\right)
$$

The value of $l_{\hat{\vec{\alpha}}_{n}}$ equals one in the linear case and becomes larger as the non-linearity around estimate $\hat{\vec{\alpha}}_{n}$ increases. Specifying $n \rightarrow \infty$ yields $l_{\hat{\vec{\alpha}}_{\infty}}$ as a measure for the convergence speed around final estimate $\hat{\vec{\alpha}}_{\infty}$ when using a least-squares Gauss-Newton estimator. An increasing value of $l_{\hat{\tilde{\alpha}}_{\infty}}$ puts a number of different measurement models into decreasing order of convergence speed.

\subsection{Assessing noise insensitivity}

Noise insensitivity of different measurement models had to be assessed by the local second-order derivatives from the Hessian matrix of the least-squares error function. The comparison of Hessian matrices from two different measurement models boils down to determining whether the error ellipsoid of one matrix encloses, intersects or is enclosed by the error ellipsoid of the other. It requires the adimensional comparison procedure from Section 3.1 to ascertain this.

$$
\left[H_{1, \hat{\vec{\alpha}}_{n}}\right]^{H_{0, \hat{\vec{\alpha}}_{n}}}=U \Gamma_{\hat{\vec{\alpha}}_{n}} U^{T}=\left[C_{0, \hat{\vec{\alpha}}_{n}}\right]^{C_{1, \hat{\tilde{\alpha}}_{n}}}
$$

The columns of matrix $U$ contain the eigenvectors and diagonal matrix $\Gamma_{\hat{\vec{\alpha}}_{n}}$ contains the corresponding eigenvalues of the Hessian matrix $H_{1}$ on a metric defined by $H_{0}$, which is equivalent to the covariance matrix $C_{0}$ on a metric defined by $C_{1}$.

Comparing two arbitrary error ellipsoids has now been simplified to a comparison of the $\left[H_{1}\right]^{H_{0}}$ ellipsoid with the unit sphere. Their intersection or mutual enclosure is determined by the (reciprocal) half-lengths of the principal axes, which are represented by the range of eigenvalue roots $\Gamma_{i i, \hat{\tilde{\alpha}}_{n}}^{\frac{1}{2}}$ of matrix $\left[H_{1}\right]^{H_{0}}$ :

$$
\mathcal{L}_{\hat{\vec{\alpha}}_{n}}=\left\langle\min _{i=1}^{m}\left(\Gamma_{i i, \hat{\vec{\alpha}}_{n}}^{\frac{1}{2}}\right), \max _{i=1}^{m}\left(\Gamma_{i i, \hat{\vec{\alpha}}_{n}}^{\frac{1}{2}}\right)\right\rangle
$$

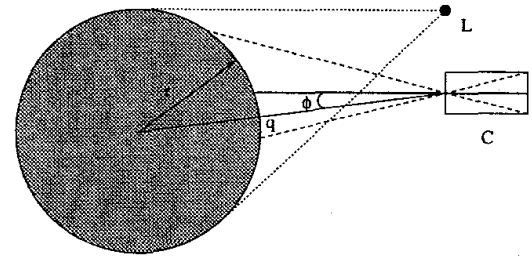

Figure 4: The $x y$-plane projection of the cylinder scene.

The positioning of unit length with respect to this range $\mathcal{L}_{\hat{\vec{\alpha}}_{n}}$ may indicate which of the $H_{0}$ and $H_{1}$ measurement models is least noise-sensitive around estimate $\hat{\vec{\alpha}}_{n}$. If all eigenvalues exceed unity, the $H_{0}$ error ellipsoid encloses the $H_{1}$ error ellipsoid, so the $H_{1}$ model provides better noise insensitivity in all directions of parameter space. The opposite holds if all eigenvalues are smaller than unity. However, if unity falls into the range $\mathcal{L}_{\hat{\tilde{\alpha}}_{n}}$, the $H_{0}$ and $H_{1}$ error ellipsoids intersect and pointing out the least noisesensitive model is depending on the viewing direction of parameter space. Making a choice based on one single direction would be equivalent to defining a norm on a Hessian matrix (see Section 3.1).

Quantitative interpretation of the roots in $\mathcal{L}_{\hat{\vec{\alpha}}_{n}}$ as well as judgement of the overall noise insensitivity in case of intersecting error ellipsoids requires knowledge from the application domain. The question whether a covariance improvement of a parameter with a certain physical dimension will outweigh a covariance worsening of another parameter with a different physical dimension can not be answered unconditionally. But realizing that the many-dimensionality of the parameter space may hinder the visualization of error ellipsoids, the root range $\mathcal{L}_{\hat{\vec{\alpha}}_{\infty}}$ still provides us with a useful tool for judging the noise insensitivity of two different measurement models around the final estimate $\hat{\vec{\alpha}}_{\infty}$. Noise insensitivity analysis of more than two measurement models generally requires the mutual comparison of each pair to get a complete overview.

\section{Modelling}

The scene model we use for the estimation of geometrical parameters consists of one solid opaque cylinder aligned with the $z$-axis of the world. Figure 4 shows a top view of such a scene. The cylinder is irradiated by a single isotropic point light source $L$. The imaging model supplies a perspective projection of the reflectance onto the CCD-elements of a pinhole line-scan camera $C$ aligned with the $x y$-plane (see e.g. Van der Heijden [10]). Figure 1 showed an example of the camera output without measurement noise. The cylinder surface consists of a plastic material, which guarantees both a significant diffuse and (off)-specular reflection component. 


\begin{tabular}{|c||c|c|c|}
\hline$\vec{\theta}$ & $=$ & $\operatorname{dim} \vec{\theta}$ & $C_{\vec{\theta}}$ \\
\hline$\vec{\theta}_{d+s}$ & $\vec{E}_{d+s}$ & 128 & $\left(\sigma_{d}^{2}+\sigma_{s}^{2}\right) I$ \\
$\vec{\theta}_{d}$ & $\vec{E}_{d}$ & 128 & $\sigma_{d}^{2} I$ \\
$\vec{\theta}_{s}$ & $\vec{E}_{s}$ & 128 & $\sigma_{s}^{2} I$ \\
$\vec{\theta}_{d \| s}$ & $\left(\begin{array}{c}\vec{E}_{d} \\
\vec{E}_{s}\end{array}\right)$ & 256 & $\left(\begin{array}{cc}\sigma_{d}^{2} I & O \\
O & \sigma_{s}^{2} I\end{array}\right)$ \\
\hline
\end{tabular}

Table 1: Measurement vectors $\vec{\theta}$ and covariances $C_{\vec{\theta}}$.

\subsection{Measurement models}

The use of separated reflection components will be studied by applying four different measurement models $\vec{\theta}(\vec{\alpha})$ in the parameter estimation process. Table 1 lists the measurement vectors produced by these models in conformity with Eq. (1). Measurement vector $\vec{\theta}_{d+s}$ contains the unseparated irradiance, $\vec{\theta}_{d}$ the diffuse component, $\vec{\theta}_{s}$ the specular component and the double-sized vector $\vec{\theta}_{d \| s}$ both reflection components in parallel. A separation algorithm has to filter these vectors from the vectorized irradiance $\vec{E}$ of the 1-D array of CCD-elements (see Figure 1).

The model approximations $\vec{\theta}\left(\hat{\vec{\alpha}}_{n}\right)$ of the measurement vector are generated by a ray-tracer. The lack of a physical scene implies that the process generating the real $\vec{\theta}$ has to be simulated by ray-tracing $\vec{\theta}(\vec{\alpha})$ and adding noise $\underline{\vec{n}}$. The measurement vectors $\vec{\theta}_{d}$ and $\vec{\theta}_{s}$ are considered to be affected by additive zero-mean Gaussian noise with no spatial correlation and with constant standard deviations $\sigma_{d}$ and $\sigma_{s}$ respectively. Thermal and fixed pattern noise in CCD-elements satisfies these properties [10]. If separation of reflection components is established by means of multiple colour or polarization channels, it may be assumed that no correlation exists between the noise in $\vec{\theta}_{d}$ and $\vec{\theta}_{s}$. This leads to the definition of the covariance matrices $C_{\vec{\theta}}$ as listed in Table 1.

\section{Experimental results}

All our performance experiments refer to the simultaneous estimation of the geometrical parameters from the scene of Figure 4. The 3-dimensional parameter vector $\vec{\alpha}$ consists of the radius $r$ of the cylinder, its distance $q$ to the camera and its angle $\phi$ with the optical axis. All other parameters in the imaging and reflectance models are assumed to be known.

Although the behaviour of a non-linear estimator varies in its parameter space, relevance is preserved when confining the analysis to a representative point. Therefore, our measurement vector $\vec{\theta}$ always originates from the irradiance profiles of Figure 1 that is assumed to be a representative image of a highlight on the cylinder surface. Instead of estimating final estimate $\hat{\vec{\alpha}}_{\infty}$ for a whole set of noise realizations, we may expect

\begin{tabular}{|c||c|c|c|c|}
\hline$\vec{\theta}(\vec{\alpha})$ & $\vec{\theta}_{d+s}(\vec{\alpha})$ & $\vec{\theta}_{d}(\vec{\alpha})$ & $\vec{\theta}_{s}(\vec{\alpha})$ & $\vec{\theta}_{d \| s}(\vec{\alpha})$ \\
\hline$l_{\hat{\alpha}_{\infty}}$ & 1.46 & 1.09 & 4.53 & 1.34 \\
\hline
\end{tabular}

Table 2: Linearity measure $l_{\hat{\tilde{\alpha}}_{\infty}}$ for different models.

\begin{tabular}{|c|c|c|c|}
\hline$\left[H_{1}\right]^{H_{0}}$ & $\overline{H_{d \| s}}$ & $H_{s}$ & $\overline{H_{d}}$ \\
\hline$\left[H_{d+s}\right]$ & $\langle\overline{\langle 0.62,0.82\rangle}$ & $\overline{\langle\langle 0.74,2.27\rangle}$ & $\overline{\langle 0.68,4.53\rangle}$ \\
\hline$\left[H_{d}\right]$ & $\langle 0.16,0.94\rangle$ & $\langle 0.16,2.77\rangle$ & \\
\hline$\left[H_{s}\right]$ & $\langle 0.34,0.99\rangle$ & & \\
\hline
\end{tabular}

Table 3: Root range $\mathcal{L}_{\hat{\bar{\alpha}}_{\infty}}$ of matrix $\left[H_{1}\right]^{H_{0}}$ for $\sigma_{d}=\sigma_{s}$.

a representative estimator performance from realization $\vec{n}=\overrightarrow{0}$. It converges to the real parameter vector $\vec{\alpha}$, which is the mean final estimate for an unbiased estimator.

\subsection{Convergence measurements}

The non-linearity measure $l_{\hat{\tilde{\alpha}}_{\infty}}$ from Section 3.2 which determines the convergence speed around final estimate $\hat{\vec{\alpha}}_{\infty}$ has been listed in Table 2 for all four measurement models $\vec{\theta}(\vec{\alpha})$. Figure 5 shows intersections of the corresponding $\left[H_{-\vec{\Delta}}\right]^{H_{+\Delta}}$ disparity ellipsoids with the principal planes of the 3-D parameter space after the co-ordinate transformation. Notice that in general the principal axes of the ellipsoids do not coincide with those planes. The diffuse component $\vec{\theta}_{d}$ will yield the best convergence speed, because its $l_{\hat{\tilde{\alpha}}_{\infty}}$ value and disparity ellipsoid are closest to unity. The specular component $\vec{\theta}_{s}$ participating in the three other measurement models definitely increases their non-linearity. These analytical results are consonant with the experimental convergence measurements reported by Glas and Van der Heijden [13].

\subsection{Noise insensitivity analysis}

The root range $\mathcal{L}_{\hat{\vec{\alpha}}_{\infty}}$ from Section 3.3 which contains the eigenvalue roots of the $\left[H_{1}\right]^{H_{0}}$ matrix around final estimate $\hat{\vec{\alpha}}_{\infty}$ has been listed in Table 3 for all pairs of measurement models. Measurement noise of the diffuse and specular reflection components is given by fixed standard deviations $\sigma_{d}=\sigma_{s}$ (see Table 1). The first column of Table 3 attributes the best noise insensitivity to measurement model $\vec{\theta}_{d \|_{s}}(\vec{\alpha})$, because it proves that on a metric defined by Hessian matrix $H_{d \| s}$ the unit sphere is enclosed by the ellipsoids of all other measurement models. Putting those three models into order of noise insensitivity is disputable for their mutual intersection that follows from the last two columns of Table 3.

Figure 6 shows principal plane intersections through the centre $\overrightarrow{\hat{\alpha}}_{\infty}$ of the error ellipsoids of all measurement models. These intersections correspond with the error ellipses of estimation problems in which two param- 

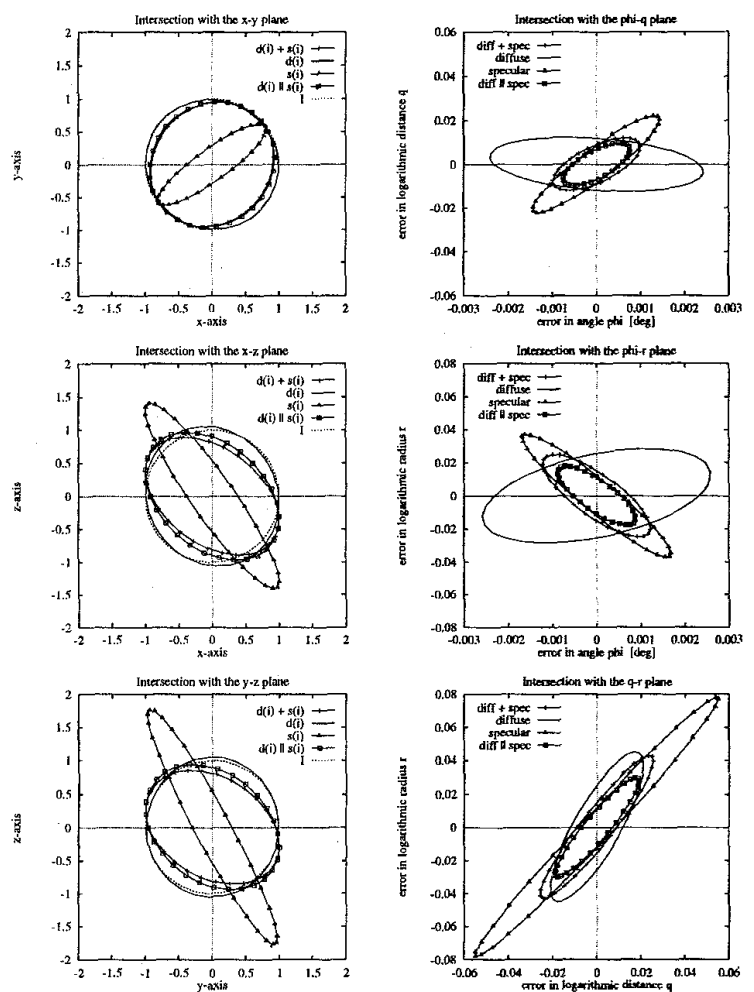

Figure 5: Principal plane intersections of the $\left[H_{-\vec{\Delta}}\right]^{H_{+} \vec{\Delta}}$ disparity ellipsoids.
Figure 6: Principal plane intersections of the $1-\sigma$ error ellipsoids for $\sigma_{d}=\sigma_{s}$.

eters of $\vec{\alpha}$ are unknown and the third one is known. Notice that in general the enclosure of a 3-D ellipsoid is not guaranteed by enclosure of its principal plane intersections. Nevertheless, these pictures confirm that measurement model $\vec{\theta}_{d \| s}(\vec{\alpha})$ yields the best noise insensitivity. Repeating the analysis for any $\sigma_{d} \neq \sigma_{s}$ will show the same superiority [13].

\section{Conclusions}

The main conclusion is that separated reflection components benefit the estimation of geometrical parameters of 3-D objects from images. The best strategy concerning the convergence properties and noise insensitivity starts estimating with the smooth diffuse component only to attain fast convergence. Modelling also the sharp specular reflectance is useful in the ultimate stage of the estimation. Change-over to a measurement vector containing the diffuse and specular components in parallel will improve the noise insensitivity of the final estimate.

Throughout this paper we have assumed uncorrelated additive Gaussian measurement noise. Only a bad separation method may cause anti-correlated noise

in diffuse and specular reflection components, that would harm noise insensitivity. Quantum noise from CCD-elements and fluctuations with respect to the reflectance model provide signal-dependent noise [10]. The least-squares estimator is not optimal in that case, but its performance may still benefit from reflection component separation.

Comparing local higher-order derivatives of the least-squares error function appears to be a useful tool to analyse the estimation performance of different measurement models. It surmounts the difficulties in visualizing many-dimensional parameter spaces and reduces the amount of experimental effort required to cover non-linearity of the model and uncertainty in its measurements. When adapting the order of the derivatives to be compared, similar methods can be applied to analyse the performance of estimators with other error criteria like least-absolute-values [9] or other iteration methods like Newton-Raphson [8].

\section{References}

[1] M.J. Korsten, "Three-Dimensional Body Parameter Estimation from Digital Images", Ph.D. Thesis, University of Twente, The Netherlands, 1989.

[2] A.J. de Graaf, M.J. Korsten, Z. Houkes, "Estimation of Position and Orientation of Objects from Stereo Images", Proc. 12. DAGM-Symposium, Mustererkennung 1990, Informatik-Fachberichte 254, Oberkochen-Aalen, Germany, pp. 348-355, 1990.

[3] D.G. Lowe, "Fitting Parameterized Three-Dimensional Models to Images", IEEE Tr. Patt. Anal. Mach. Intell, vol. 13, No. 5, pp. 441-450, May 1991.

[4] R. Gershon, "The Use of Color in Computational Vision", Ph.D. Thesis, University of Toronto, Canada, 1987.

[5] G.J. Klinker, "A Physical Approach to Color Image Understanding", Ph.D. Thesis, Carnegie Mellon University, USA, 1988.

[6] L.B. Wolf, T.E. Boult, "Constraining Object Features Using a Polarization Reflection Model", IEEE Tr. Patt. Anal. Mach. Intell., vol. 13, no. 7, pp. 635-657, July 1991.

[7] S.K. Nayar, X.S. Fang, T. Boult, "Removal of Specularities Using Color and Polarization", Proc. IEEE Comp. Vis. Patt. Recogn., New York, USA, pp. 583-590, 1993.

[8] H.W. Sorensen, Parameter Estimation, Principles and Problems, Control and Systems Theory, vol. 9, Marcel Dekker, New York, USA, 1980.

[9] A. Tarantola, Inverse Problem Theory, Methods for Data Fitting and Model Parameter Estimation, Elsevier, Amsterdam, The Netherlands, 1987.

[10] F. van der Heijden, Image Based Measurement Systems, Object Recognition and Parameter Estimation, John Wiley \& Sons, Chicester, UK, 1994.

[11] S.K. Nayar, K. Ikeuchi, T. Kanade, "Surface Reflection: Physical and Geometrical Perspectives", IEEE Tr. Patt. Anal. Mach. Intell., vol. 13, No. 7, pp. 611-634, July 1991.

[12] K. Torrance, E. Sparrow, "Theory for Off-Specular Reflection from Roughened Surfaces", J. Opt. Soc. Am. A, vol. 57, no. 7, pp. 1105-1114, July 1967.

[13] J.C. Glas, F. van der Heijden, "The Use of Separated Reflection Components in Estimating Geometrical Parameters of Curved Surface Elements", accepted at 5th IEE Conf. Image Proc. Appl., Edinburgh, UK, July 1995. 